Pacific Journal of Mathematic 


\title{
HOMEOMORPHISMS OF LONG CIRCLES WITHOUT PERIODIC POINTS
}

\author{
RICHARD FreimaN
}

\begin{abstract}
Markley characterized those minimal sets arising from a homeomorphism of the circle without periodic points. The removal of the condition that guarantees the space be metric yields a larger class of minimal sets which can still be embedded in a circle-like object. A $\{$ long $\}$ sshort $\}$ circle is a compact connected \{non-metric Hausdorff \{metric\} space which has no cut points, but which is disconnected by the removal of any two points. A circle is either a long or short circle. Theorem: A compact Hausdorff minimal cascade can be embedded in a homeomorphism of a circle without periodic points if and only if it is proximally equicontinuous over the circle and $P^{\prime} \subseteq \Delta$, where $P$ is the proximal relation.
\end{abstract}

Homeomorphisms of the circle were first studied by Poincare [12]. They may be divided into two classes-transitive and intransitiveaccording to whether or not there is a dense orbit. Each such homeomorphism has a unique minimal set and Markley has characterized those minimal sets which could arise from such a homeomorphism. Dropping his cardinality restriction on the proximal relation leads to the idea of embedding such minimal sets in "long" (i.e., non-metric) circles. In order to obtain necessary as well as sufficient conditions for a minimal set to arise from a homeomorphism of a "long" circle without periodic points such homeomorphisms are investigated. We shall begin by defining "long" circles and associated spaces, describing some of their properties and giving some examples. A study of homeomorphisms without periodic points of "long" circles follows. We then proceed to state and prove the main theorem of this paper.

A linearly ordered set $(X,<)$ together with the order topology is a linearly ordered topological space (LOTS). A pair of disjoint nonempty complementary subsets $A, B$ of $X$ such that every element in $A$ precedes every element in $B$ is a cut and is denoted $[A \mid B]$. If in a cut $[A \mid B] A$ contains its least upper bound (LUB) and $B$ contains its greatest lower bound (GLB), we say $X$ has a jump. If $A$ does not have its LUB and $B$ does not have its GLB, we say $X$ has a gap. If $X$ has no gaps, then $X$ is order-complete. If $X$ is order complete, then every nonempty set with upper bound has a LUB. A subset of an order-complete LOTS is compact if and only if it is closed and order-bounded and it is connected if and only if it is order-complete and without jumps. [9, Problems 1I, 5C.] A connected LOTS is therefore locally connected and locally compact. A point $x$ in a topological 
space $X$ is called a cut point if $X-\{x\}$ is not connected. Otherwise, it is a non-cut point.

It is immediate that a LOTS which is order-complete without jumps and with first and last elements is a compact connected Hausdorff space with exactly two non-cut points. The additional condition of metrizability would imply the space be an arc, i.e., a homeomorph of a closed interval of reals. This suggests the following definitions.

For the sake of brevity we shall refer to a simple closed curve as a circle. A long $\{$ arc $\}$ \{circle $\}$ is a non-metric compact connected Hausdorff space which \{has exactly two non-cut points\} no cut points, but which disconnected by the removal of any two points\}. A long Cantor set is a non-metric compact totally disconnected Hausdorff space. We shall precede the word \{arc\} \{circle\} \{Cantor set\} with the adjective "short" in order to refer to the usual (i.e., metric) \{arc\} \{circle\} \{Cantor set\} and reserve the word $\{$ arc $\}$ \{circle\} \{Cantor set\} for a space which may be either a long or short \{arc\} \{circle\} \{Cantor set\}.

Let $\Lambda=\{(x, y): 0 \leqq x, y \leqq 1\}$ be the unit square with lexicographic order, i.e., $\left(x_{1}, y_{1}\right)$ precedes $\left(x_{2}, y_{2}\right)$ if and only if $x_{1}<x_{2}$ or $x_{1}=x_{2}$ and $y_{1}<y_{2}$. Then $\Lambda$ is an arc. It is first countable but not separable (hence, it is not metrizable) and is therefore a long arc [9, problem 5J].

If we identify points at the same height on the two vertical edges of $\Lambda$ we obtain a cylinder $\Sigma$ which may be described analytically by $\Sigma=S \times I$, where $S$ is the group of complex numbers of modulus one and $I=[0,1]$. Then $\Sigma$ is a compact connected Hausdorff space without cut points which is disconnected by the removal of any two points. It is first countable but not separable and so is a long circle.

It is known that an arc is short if and only if it is separable [7], [10]. It follows that a circle is short if and only if it is separable. The corresponding result for Cantor sets is as follows: A Cantor subset of a connected LOTS is a short Cantor set if and only if the number of jumps is countable [13, Chapter 1, Theorem 20].

Unlike the topological characterizations of short arc, circle, and Cantor sets there are nonhomeomorphic long arcs, circles and Cantor sets. If the first uncountable ordinal, $\Omega$, is added to the "long line" [6, page 55] as its last element, it becomes a long arc, $\Lambda^{\prime}$. The last element does not have a countable base for its neighborhoods and so the spaces $\Lambda$ and $\Lambda^{\prime}$ are not homeomorphic. If we identify the first and last elements of this space we obtain a long circle $\Sigma^{\prime}$ which is not homeomorphic to $\Sigma$. If we use Cantor ternary sets instead of open intervals to fill in the jumps between successive ordinals in the formation of the "long line" we obtain a long Cantor set which is not homeomorphic to any Cantor subset of $\Lambda$. In the opposite direction it will be useful to observe that long ares share the fixed point property in common with the short arc. Further, although some points may 
not have a countable basis for their neighborhoods it is true that any point of a separable subset $S$ of a LOTS $X$ will have a countable basis for its' neighborhoods (in the order topology of $X$ ). [8, Theorems $2,8,2.10]$.

If $A$ is a subset of a topological space, we denote its set of accumulation points by $A^{\prime}$ and the closure of $A$ by $\bar{A}$.

We are now in a position to investigate homeomorphisms of a long circle without periodic points and to prove results similar to some of those in van Kampen [14] and Markley [11] for homeomorphisms of a short circle without periodic points. It should be noted that in contrast to the case of the short circle a long circle cannot have a transitive homeomorphism since no countable set can be dense. Further, some long circles do not admit homeomorphisms without periodic points. The long circle, $\Sigma^{\prime}$, is one such example. In the observation following the proof of Theorem 4 we will discern something of the structure of long circles which do admit such homeomorphisms.

If $X$ is a topological space and $\phi: X \rightarrow X$ is a homeomorphism, then $(X, \phi)$ is called a discrete flow or cascade. The orbit of a point $x$ under $\phi$, denoted $\boldsymbol{O}(x, \phi)$, is $\left\{\phi^{n}(x): n\right.$ is an integer $\}$. If $\overline{\boldsymbol{O}(x, \phi)}=X$ for each $x \in X$, then $(X, \phi)$ is said to be a minimal discrete flow or minimal cascade. It may also be referred to as a minimal set. If $Q$ is a concept or relation defined for a transformation group $(X, \phi)$ and there is a need to specify the space to which $Q$ applies we attach the symbol of the space to $Q$, e.g., $Q(X)$ or $Q_{X}$. The points $x, y \in X$ are said to be proximal if whenever $\alpha \in U$, the uniformity of $X$, there is an integer $n$ such that $\left(\phi^{n}(x), \phi^{n}(y)\right) \in \alpha$. The proximal relation is denoted by the letter $P$. If $x$ and $y$ are not proximal, they are said to be distal.

If $(Y, \psi)$ and $(Z, \pi)$ are isomorphic we denote this by $(Y, \psi) \cong$ $(Z, \pi)$. By $(Y, \psi) \simeq(Z, \pi)$ we denote that there is a homomorphism from $(Y, \psi)$ onto $(Z, \pi)$. We use $S$ to denote the set of complex numbers of modulus one. An element $g \in S$ is said to be a generator of $S$ if $\left\{g^{n}: n\right.$ is an integer $\}$ is dense in $S$. Define $M_{g}: S \rightarrow S$ by $M_{g}(z)=g z$. Then $\left(S, M_{g}\right)$ is an equicontinuous minimal set. A transformation group $(Y, \psi)$ is proximally equicontinuous if $P$ is closed and the quotient

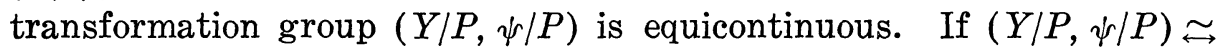
$\left(S, M_{g}\right)$ then $(Y, \psi)$ is said to be proximally equicontinuous over the short circle.

THEOREM 1. Let $S^{*}$ be a long circle and let $\phi$ be a homeomorphism of $S^{*}$ without periodic points. There exists a Cantor set $C$ which is the only minimal set under $\phi$ and such that $\overline{\boldsymbol{O}(x, \phi)}-\boldsymbol{O}(x, \phi)=C$ for all $x \in S^{*}-C$.

Proof. There exists a minimal set $C$. If $\operatorname{int}(C) \neq \varnothing$, then $C$ is 
an open and closed subset of $S^{*}$, therefore $C=S^{*}$ which contradicts $S^{*}$ not separable. Hence, $\operatorname{int}(C)=\varnothing$. Because there are no periodic points $C$ is perfect. The complement of $C$ in $S^{*}$ is a union of disjoint open intervals. If $J$ is a complementary interval, then $\phi^{n}(J)$ is a complementary interval and $J \cap \phi^{n}(J)=\varnothing$ for $n \neq 0$. If $J \cap \phi^{n}(J) \neq \varnothing$ for some $n \neq 0$, then $\phi^{n}(J)=J$. This same identity holds for the closure of complementary intervals which then will have a fixed point. This in turn implies $\phi$ has a periodic point. Let $x \in J \subseteq S^{*}-C$. Then $\boldsymbol{O}(x, \phi)$ is a countable discrete set in $S^{*}-C$. Since $\overline{\boldsymbol{O}(x, \phi)}-\boldsymbol{O}(x, \phi) \subseteq C$ and contains a minimal set, $\overline{\boldsymbol{O}(x, \phi)}-\boldsymbol{O}(x, \phi)=C$ and $C$ is the only minimal set in $S^{*}$.

Corollary 1. Each point in the complement of $C$ is doubly asymptotic to $C$.

Proof. Let $x \in S^{*}-C$ and let $U$ be the unique uniformity of $S^{*}$. If $x$ is not doubly asymptotic to $C$, then there is a sequence $\left\{n_{i}\right\}$ with $\left|n_{i}\right| \rightarrow \infty$ such that $\left\{\phi^{n_{i}}(x)\right\} \cap C \alpha=\varnothing$ for some $\alpha \in U$. This implies the existence of $y \in \overline{\boldsymbol{O}(x, \phi)}-\boldsymbol{O}(x, \phi)$ such that $y \notin C$, which is impossible. Thus, $x$ is doubly asymptotic to $C$.

Theorem 2. Let $S^{*}$ be a long circle, let $\phi: S^{*} \rightarrow S^{*}$ be a homeomorphism without periodic points and let $C$ be the unique Cantor minimal set in $S^{*}$. Then $(x, y) \in\left\{P\left(S^{*}\right)\right\}\{P(C)\}$ if and only if $x=y$ or $\{x$ and $y$ are in the same closed complementary interval $\}\{x$ and $y$ are endpoints of the same complementary interval $\}$. Thus, $\left\{P\left(S^{*}\right)\right\}$ $\{P(C)\}$ is an invariant closed equivalence relation and $\left\{\left(S^{*}, \phi\right)\right\}\{(C, \phi / C)\}$ is proximally equicontinuous over the short circle.

Proof. Define the relation $R$ on $S^{*}$ by $(x, y) \in R$ if and only if $x=y$ or $x$ and $y$ are in the same closed complementary interval. It is easily seen to be an invariant equivalence relation. We show $R \cong P\left(S^{*}\right), R$ is closed and $\left(S^{*} / R, \phi / R\right) \cong\left(S, M_{g}\right)$ for some generator $g \in S$. These imply $P\left(S^{*}\right)=R$.

We shall denote intervals of $S^{*}$, open, closed, etc. by $(x, y),[x, y]$, etc. Whether $(x, y)$ is an open interval or an ordered pair will be clear from the context.

Denote by $(a, b)$ an interval of $S^{*}$ proceeding from $a$ to $b$ in the clockwise direction. Let $\left(x_{0}, y_{0}\right)$ be a complementary interval. Let $\left\{n_{i}\right\}$, $i \geqq 0$, be a sequence with $n_{0}=0$ and $\left|n_{i}\right| \rightarrow \infty$ such that $\phi^{n_{i}}\left(x_{0}\right) \rightarrow \bar{x}$ and $\phi^{n_{i}}\left(y_{0}\right) \rightarrow \bar{y}$. Since $\bar{x}, \bar{y}$ are in $C$, a separable subset, such sequences exist. Denote $\phi^{n_{i}}\left(x_{0}\right)$ by $x_{i}$ and $\phi^{n_{i}}\left(y_{0}\right)$ by $y_{i}$. We may assume without loss of generality that $\bar{y} \notin\left(y_{0}, \bar{x}\right)$, i.e., when we proceed from $y_{0}$ in a clockwise direction we do not reach $\bar{y}$ before $\bar{x}$. Since neighborhoods 
of points on a long circle are connected LOTS (i.e., intervals) we shall think of these neighborhoods as having a linear order induced by the clockwise direction on the circle. Thus, we may speak of being to the left or right of a point. Since a subsequence of $\left\{x_{i}\right\}$ must converge to $\bar{x}$ from one side or the other, we shall assume that it converges from the right. If it converges from the left the proof is slightly easier due to the order we have assumed for $x_{0}, y_{0}, \bar{x}$, and $\bar{y}$. To avoid too complicated notation we shall denote the subsequence by $\left\{x_{i}\right\}$ and we shall assume, without loss of generality, that $\left\{x_{i}\right\}$ is monotonically decreasing. Complementary intervals being disjoint, $\left\{y_{i}\right\}$ also is a monotonically decreasing sequence which converges to $\bar{y}$. Since $\bar{y}$ is not in any complementary interval, we conclude all the intervals $\left(x_{i}, y_{i}\right)$ (or $\left(y_{i}, x_{i}\right)$ ) are to the right of $\bar{y}, x_{i} \downarrow \bar{y}$ and $\bar{x}=\bar{y}$. Clearly, for all $z \in\left(x_{0}, y_{0}\right), \phi^{n_{i}}(z) \rightarrow \bar{x}=\bar{y}$. Thus, $R \subseteq P\left(S^{*}\right)$.

If we use a sequence of endpoints of complementary intervals rather than the iterates of a single complementary interval, we may mimic the proof of $R \subseteq P\left(S^{*}\right)$ to derive a proof that $R^{\prime} \subseteq \Delta$ and therefore, that $R$ is closed.

$S^{*} / R$ is compact, connected, remains connected upon the removal of a single point but is disconnected by the removal of two points. The proof of this is the same as that for the case of a short circle and depends only upon the non-metric properties of $S^{*}$ and the nature of $R$ [14]. $\left(S^{*} / R, \phi / R\right)$ is a minimal set. Therefore, $S^{*} / R$ is separable and metrizable. Thus, $\left(S^{*} / R, \phi / R\right) \approx\left(S, M_{g}\right)$ for some generator $g \in S$. It follows that $P\left(S^{*}\right) \subseteq R$ and therefore, $P\left(S^{*}\right)=R$.

The companion remarks about $P(C)$ and $(C, \phi / C)$ follow from slight modifications of the above remarks.

This result as well as the next two were obtained for the short circle by making use of its usual metric. Since the arc length of the unit circle is $2 \pi$ the sum of the lengths of a sequence of disjoint intervals must converge which implies that the sequence of lengths converges to 0 .

Corollary 2. The proximal relation of $(C, \phi / C)$ is given by $(x, y) \in P(C)$ if and only if $x=y$ or $x$ and $y$ are endpoints of some complementary interval. Thus $P(C)$ is an invariant closed equivalence relation and $(C, \phi / C)$ is a proximally equicontinuous minimal set over the short circle.

THEOREM 3. Let $\phi$ be a homeomorphism of a long circle $S^{*}$ without periodic points. Then, $P^{\prime}\left(S^{*}\right) \subseteq \Delta\left(S^{*}\right)$ and $P^{\prime}(C) \subseteq \Delta(C)$.

Proof. In the proof of Theorem 2 we showed $R^{\prime} \subseteq \Delta$. Hence, 
$P^{\prime}\left(S^{*}\right) \subseteq \Delta\left(S^{*}\right)$. Since $P(C)=P\left(S^{*}\right) \cap(C \times C)$, we have $P^{\prime}(C) \subseteq \Delta(C)$.

We now come to the main theorem of this paper.

If $\left(X_{\alpha}, \psi_{\alpha}\right)(\alpha \in I)$ is a collection of cascades we define the product cascade $(X, \psi)$ by $X=\prod_{\alpha \in I} X_{\alpha}$ and $\psi\left(\left(x_{\alpha}\right)\right)=\left(\psi_{\alpha}\left(x_{\alpha}\right)\right)$. A property $P$ of cascades is said to be admissible if (i) there is at least one minimal set having the property and (ii) if $\left(X_{\alpha}, \psi_{\alpha}\right)(\alpha \in I)$ is a collection of minimal cascades, each of which satisfies $P$, and if $M$ is a minimal subset of the product cascade, then $(M, \psi / M)$ satisfies $P$. This is a specialization of the definition in [2]. We shall have occasion to speak of a property as if it were the collection of sets satisfying the property, e.g., we shall say $(X, \psi) \in P$ instead of $(X, \psi)$ satisfies $P$. If a collection of cascades is admissible, then there is a universal minimal cascade

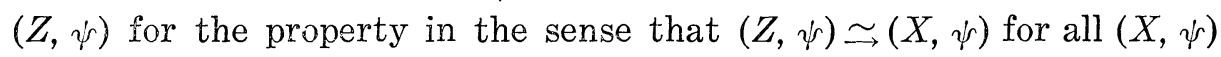
which satisfy the property. Such a minimal set is unique up to isomorphism.

Define the property (collection) of minimal cascades $P_{g}$ ( $g$ is a generator of $S)$ as follows: $(X, \psi) \in P_{g}$ if and only if $(X, \psi)$ is proximally equicontinuous over the short circle and $P^{\prime} \subseteq \Delta$. This is an admissible property and the universal minimal for $P_{g}$ is the Ellis minimal set $\left(\boldsymbol{E}, \widetilde{M}_{g}\right)$ [5]. This minimal set is defined in [4], but we shall use the description of it in Section 2 of [5]. It is a locally almost periodic regular minimal set.

In [11] Markely proved the following theorem: Let $(X, \phi)$ be a minimal cascade where $X$ is a compact Hausdorff space. Then $(X, \phi)$ can be embedded in some $(S, \psi)$ where $\psi$ has no periodic points if and only if $P^{\prime} \subseteq \Delta, P-\Delta$ is countable and $(X, \phi)$ is proximally equicontinuous over the short circle. It appears that the condition " $P-\Delta$ is countable" in the hypothesis of the theorem is needed only to insure the minimal set $(X, \phi)$ is metric so that it may be (embedded as) a subspace in $S$. Indeed, Markley remarks that it may be replaced by " $X$ is metric". One may hope therefore to drop this countability restriction and extend the theorem to the class $P_{g}$, maintaining the embedding in a non-metric object which could be a short circle when the restriction holds.

THEOREm 4. Let $(X, \phi)$ be a minimal cascade where $X$ is a compact Hausdorff space. Then $(X, \phi)$ can be embedded in some $\left(\Sigma^{*}, \Phi^{*}\right)$ where $\Sigma^{*}$ is a circle and $\Phi^{*}: \Sigma^{*} \rightarrow \Sigma^{*}$ is a homeomorphism without periodic points if and only if $(X, \phi) \in P_{g}$ for some generator $g \in S$.

REMARK. We can be more specific about the circle $\Sigma^{*}$ in which $(X, \phi)$ can be embedded. It can be obtained as a certain kind of decomposition space of the long circle $\Sigma$. 
The proof will be preceded by a series of propositions. We shall use the cylinder picture of $\Sigma$, i.e., $\Sigma=S \times I=\{(z, r): z \in S, 0 \leqq r \leqq 1\}$. For a generator of $g \in S$ define the function $\mu_{g}: S \times I \rightarrow S \times I$ by $\mu_{g}((z, r))=(g z, r)$ where $g z$ is complex multiplication. It is a homeomorphism of $\Sigma$ without periodic points which spins each horizontal short circle and therefore the cylinder by the amount arg $(g)$. Thus, $\left(\Sigma, \mu_{q}\right)$ is a cascade.

We let $\left(\mu_{g}\right)^{n}=\left(\mu_{g^{n}}\right)$ be $\mu_{g}$ iterated $n$ times and define $\Sigma\left(r_{1}, r_{2}, \cdots\right.$, $\left.r_{n}\right)=\left\{(z, r): r=r_{i}, i=1,2, \cdots, n\right\}$.

Proposition 1. The only minimal set of $\left(\Sigma, \mu_{g}\right)$ is $\Sigma(0,1)$. For $(z, r) \notin \Sigma(0,1)$ we have $(z, r)$ doubly asymptotic to $\Sigma(0,1)$ and $\overline{\boldsymbol{O}\left((z, r), \mu_{g}\right)}-$ $\boldsymbol{O}\left((z, r), \mu_{g}\right)=\Sigma(0,1)$. Furthermore, the points $\{(z, r): r \in I\}$ are uniformly doubly asymptotic to each other and to $\Sigma(0,1)$ and therefore the proximal relation of $\left(\Sigma, \mu_{g}\right)$ is defined by $\langle(z, r),(w, s)\rangle \in P(\Sigma)$ if and only if $z=w$.

Proof. Since $\mu_{g}[\Sigma(r)]=\Sigma(r)$ we have $\boldsymbol{O}\left((z, r), \mu_{g}\right) \subseteq \Sigma(r)$. The orbit $\boldsymbol{O}\left((z, r), \mu_{g}\right)$ is a countable dense (in the usual topology of $\left.S\right)$ subset of $\Sigma(r)$ and its set of accumulation points is $\Sigma(0,1)$. Thus, $\Sigma(0,1)$ is the only minimal set of $\left(\Sigma, \mu_{g}\right)$ and every point of $\Sigma$ other than those of $\Sigma(0,1)$ is doubly asymptotic to $\Sigma(0,1)$. It is equally clear that all points of $\{(z, r): r \in I\}$ are uniformly doubly asymptotic to each other and to $\Sigma(0,1)$ and that $P(\Sigma)$ is as described.

To avoid too complicated notation we shall not indicate that $\mu_{g}$ is restricted to $\Sigma(0,1)$. Which space $\mu_{g}$ is acting on will be clear from the context.

Proposition 2. For each generator $g \in S$ we have $\left(\Sigma(0,1), \mu_{g}\right) \widetilde{\leftrightarrow}$ $\left(\boldsymbol{E}, \widetilde{M}_{g}\right)$. Thus, $\left(\boldsymbol{E}, \widetilde{M}_{g}\right)$ is embedded as a compact, perfect, nowhere dense set in $\left(\Sigma, \mu_{g}\right)$.

Proof. Obvious. See description of $\boldsymbol{E}$ in Section 2 of [5].

Let $A \leqq S$. Define $R(A)$ in $\Sigma \times \Sigma$ by $\left\langle\left(z_{1}, r_{1}\right),\left(z_{2}, r_{2}\right)\right\rangle \in R(A)$ if and only if (i) $z_{1}=z_{2}$ and $r_{1}=r_{2}$ or (ii) $z_{1}=z_{2} \in A$, i.e., we identify all points on the same vertical line segment if their first coordinate is in $A$. Then $R(A)$ is a closed equivalence relation. The proof of the next proposition is straight forward.

Proposition 3. The space $\Sigma / R(A)$ is a circle and if $\mu_{g}(A)=A$, the map $\mu_{g} / R(A)$ is a homeomorphism without periodic points.

If enough intervals are "squashed down", then $\Sigma / R(A)$ is homeo- 
morphic to $S$. Once again we shall not introduce further notation to distinguish between $\mu_{g} / R(A)$ acting on $\Sigma / R(A)$ or on the image of $\Sigma(0,1)$ in $\Sigma / R(A)$ which we shall denote by $\Sigma(0,1) / R(A)$.

Proposition 4. If $S-A$ is countable, then $\Sigma / R(A)$ is a short circle and $\Sigma(0,1) / R(A)$ is a short Cantor set.

Proof. Think of the decomposition space $\Sigma / R(A)$ as a circle with "fibers" only over those points not in $A$, the other "fibers" being collapsed down to their "base points". Thus, in denoting the points of $\Sigma / R(A)$ we adopt the notation: for $z \notin A$, the $R(A)$-equivalence class of $(z, r)$ is $\{(z, r)\}$ which we denote $(z, r)$; for $z \in A$, the $R(A)$-equivalence class of $(z, r)$ is $\{(z, s): s \in I\}$ which we denote $(z, 0)$. For each $z \notin A$, let $D_{z}$ be a countable dense subset of $\{(z, r): r \in I\}$ and let $D_{0}$ be a countable dense subset of $\{(z, 0): z \in S\}$. Then, $D=\left(\bigcup_{z \notin A} D_{z}\right) \cup D_{0}$ is a countable dense subset of $\Sigma / R(A)$ which is separable and therefore a short circle. For $z \notin A$ we may regard the space obtained by removing the open interval $((z, 0),(z, 1))$ from $\Sigma / R(A)$ as a connected LOTS. Then $\Sigma(0,1) / R(A)$ is a Cantor subset of it with only countably many jumps.

Proposition 5. For each invariant closed equivalence relation $R_{E} \subseteq P_{E}$ there is a corresponding invariant closed equivalence relation $R_{\Sigma} \subseteq P_{\Sigma}\left(R_{\Sigma}=R(A)\right.$ with $\mu_{g}(A)=A$ for some $\left.A \subseteq S\right)$ such that

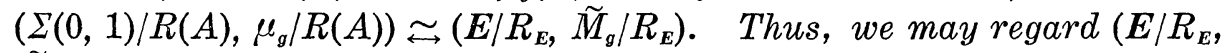
$\widetilde{M}_{g} / R_{E}$ ) embedded (as a minimal set) in $\left(\Sigma / R_{\Sigma}, \mu_{g} / R_{\Sigma}\right)$.

Proof of Theorem 4. Suppose $(X, \phi)$ is embedded in $\left(\Sigma^{*}, \Phi^{*}\right)$ where $\Phi^{*}$ is a homeomorphism without periodic points of the circle $\Sigma^{*}$. By Theorems 2 and $3(X, \phi) \in P_{g}$ for some generator $g \in S$. Conversely, suppose $(X / P, \phi / P) \cong\left(S, M_{g}\right)$ for some generator $g \in S$ and $P^{\prime} \subseteq \Delta$. There is an invariant closed equivalence relation $R_{E} \subseteq P_{E}$ such that $\left(E / R_{E}, \widetilde{M}_{g} / R_{E}\right) \approx(X, \phi)$ by Theorem 2.1 of [5]. Now apply Proposition 5 .

Observation. The proof provides a description of those circles which admit homeomorphisms without periodic points.

It follows that the long circle in which $(X, \phi)$ may be embedded is not unique.

COROLlaRy 3. Under the hypothesis in Theorem $4 \Sigma^{*}$ can be a short circle if and only if $P_{X}-\Delta_{X}$ is countable. Otherwise, it can be obtained as a long circle of a particular form.

Proof. Suppose $P_{X}-\Delta_{X}$ is countable. Theorem 2.1 of [5] enables us to conclude that the set of intervals in $\Sigma / R_{\Sigma}$ which are not "squashed 
down" is countable. Thus, $\Sigma / R_{\Sigma}$ is a short circle. Conversely, if $\Sigma^{*}$ is a short circle, it can contain no more than a countable number of disjoint open intervals. If $P_{X}-\Delta_{X}$ is uncountable, $\Sigma^{*}=\Sigma / R_{\Sigma}$ will contain uncountable many disjoint intervals that are not "squashed down" and will therefore be non-metric.

REMARK. The first part of this corollary is the theorem of Markley alluded to earlier. The proof given here is consistent with developments in this paper, but is not independent of Markley's work since Theorem 2.1 of [5] made use of his theorem.

CoRollary 4. Under the hypotheses in Theorem $4 X$ can be embedded as a short Cantor set if and only if $P_{X}-\Delta_{X}$ is nonempty and countable. If $P_{X}-\Delta_{X}$ is uncountable, it will be embedded as a long Cantor set and its complement in $\Sigma^{*}$ will be the union of uncountably many disjoint open intervals.

COROLlaRY 5. Under the hypotheses in Theorem $4(X, \phi) \approx\left(S, M_{g}\right)$ for some generator $g \in S$ if and only if $P_{X}-\Delta_{X}$ is empty. (This is the transitive case first studied in [12].)

COROLlary 6. Under the hypotheses in Theorem 4 the points in the closure of any complementary interval are uniformly doubly asymptotic to each other and to $X$ (considered as a subspace of $\Sigma^{*}$ ) and for any $y \in \Sigma^{*}-X, \overline{\boldsymbol{O}\left(y, \Phi^{*}\right)}-\boldsymbol{O}\left(y, \Phi^{*}\right)=X$. The proximal relation $P^{*}$ of $\left(\Sigma^{*}, \Phi^{*}\right)$ is given by: $(x, y) \in P^{*}$ if and only if (i) $x=y$ or (ii) $x$ and $y$ are in the closure of a complementary interval; and the proximal relation $P$ of $(X, \phi)$ is given by: $(x, y) \in P$ if and only if (i) $x=y$ (ii) $x$ and $y$ are endpoints of a complementary interval when $X$ is considered as a subspace of $\Sigma^{*}$.

COROLlary 7. Under the hypotheses in Theorem 4 each point of $X$ is a locally almost periodic of $\left(\Sigma^{*}, \Phi^{*}\right)$ when $X$ is considered as a subspace of $\Sigma^{*}$.

We are now able to add another equivalent condition to those of Theorem 4.4 in [5].

THEOREM 5. Let $(X, \dot{\phi})$ be a proximally equicontinuous regular minimal set over the short circle. Then $(X, \phi) \approx\left(E, M_{g}\right)$ if and only if $(X, \dot{\phi})$ can be embedded in $\left(\Sigma, \mu_{g}\right)$ for some generator $g \in S$.

Proof. If $(X, \phi)$ is embedded in $\left(\Sigma, \mu_{g}\right)$, then $R_{\Sigma}=\Delta_{z}$ implying

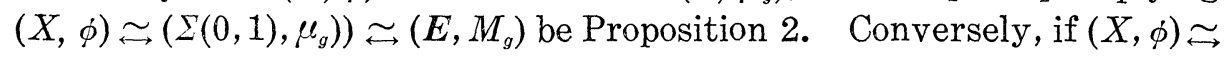


$\left(E, M_{g}\right)$, then $(X, \phi) \in P_{g}$ by Proposition 2.1 of [5]. By Theorem 4 we can embed $(X, \phi)$ in $\left(\Sigma / R_{\Sigma}, \mu_{g} / R_{\Sigma}\right)$ with $R_{\Sigma} \subseteq P_{\Sigma}$. It is clear, in fact, that $R_{\Sigma}=\Delta_{\Sigma}$. Thus, $(X, \phi)$ is embedded in $\left(\Sigma, \mu_{g}\right)$.

I would like to thank Professor Nelson Markley for conversations helpful in writing this paper.

\section{REFERENCES}

1. J. Auslander, Endomorphisms of minimal sets, Duke Math. J., 30 (1963), 605-614.

2. - Regular minimal sets I, Trans. Amer. Math. Soc., 123 (1966), 469-479.

3. G. Birkhoff, Lattice Theory, 3rd ed., Amer. Math. Soc., Providence, 1967.

4. R. Ellis and W. H. Gottschalk, Homomorphisms of transformation groups, Trans. Amer. Math. Soc., 94 (1960), 258-271.

5. R. Freiman, Regular minimal sets over the circles and the Ellis minimal set, to appear in Math. Systems Theory.

6. Hocking and Young, Topology, Addison-Wesley, Reading, 1961.

7. F. B. Jones, Concerning certain linear abstract spaces and simple continuous curves, Bull. Amer. Math. Soc., 45 (1939), 623-628.

8. I. Juhász et. al., Cardinal functions in topology, Math. Centre Tract 6, Amsterdam. 9. J. L. Kelley, General Topology, D. Van Nostrand, Princeton, 1955.

10. D. J. Lutzer and H. R. Bennett, Separability, the countable chain condition and the Lindelöf property in linearly orderable spaces, Proc. Amer. Math. Soc., 23 (1969), 664667.

11. N. G. Markley, Homeomorphisms of the circle without periodic points, Proc. London Math. Soc., (3) 20 (1970), 688-698.

12. H. Poincaré, Sur les courbes défines par les equations differentielles, J. Math. Pures Appl., (4) 1 (1885), 167-244.

13. J. van Dalen, Finite products of locally compact ordered spaces, rapp. nr. 26 (1972), Wisk. Sem. Vrije Univ., Amsterdam.

14. E. R. van Kampen, The topological transformations of a simple closed curve into itself, Amer. J. Math., 57 (1935), 142-152.

Received July 20, 1972 and in revised form August 17, 1973.

UNIVERSITY OF MARYLAND BALTIMORE COUNTY 


\section{PACIFIC JOURNAL OF MATHEMATICS}

\section{EDITORS}

RICHARD ARENS (Managing Editor) University of California

Los Angeles, California 90024

R. A. BeaUmont

University of Washington Seattle, Washington 98105
J. DUGUNDJI*

Department of Mathematics University of Southern California Los Angeles, California 90007

D. Gilbarg and J. Milgram Stanford University

Stanford, California 94305

\section{ASSOCIATE EDITORS}

E. F. BECKENBACH

B. H. NeUmanN

F. WOLF

K. YoSHIDA

\section{SUPPORTING INSTITUTIONS}

\section{UNIVERSITY OF BRITISH COLUMBIA CALIFORNIA INSTITUTE OF TECHNOLOGY UNIVERSITY OF CA.LIFORNIA MONTANA STATE UNIVERSITY UNIVERSITY OF NEVADA NEW MEXICO STATE UNIVERSITY OREGON STATE UNIVERSITY UNIVERSITY OF OREGON OSAKA UNIVERSITY}

UNIVERSITY OF SOUTHERN CALIFORNIA STANFORD UNIVERSITY UNIVERSITY OF TOKYO UNIVERSITY OF UTAH WASHINGTON STATE UNIVERSITY UNIVERSITY OF WASHINGTON AMERICAN MATHEMATICAL SOCIETY NAVAL WEAPONS CENTER

* C. R. DePrima California Institute of Technology, Pasadena, CA 91109, will replace J. Dugundji until August 1974. 


\section{Pacific Journal of Mathematics}

\section{Vol. 50, No. $1 \quad$ September, 1974}

Gail Atneosen, Sierpinski curves in finite 2-complexes.............. 1

Bruce Alan Barnes, Representations of $B^{*}$-algebras on Banach spaces .... 7

George Benke, On the hypergroup structure of central $\Lambda(p)$ sets ....... 19

Carlos R. Borges, Absolute extensor spaces: a correction and an

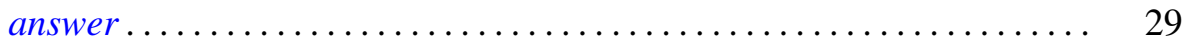

Tim G. Brook, Local limits and tripleability .................. 31

Philip Throop Church and James Timourian, Real analytic open maps .... 37

Timothy V. Fossum, The center of a simple algebra ............... 43

Richard Freiman, Homeomorphisms of long circles without periodic

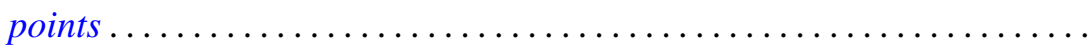

B. E. Fullbright, Intersectional properties of certain families of compact

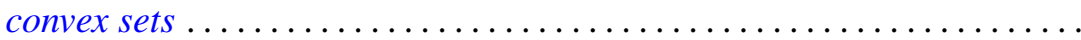

Harvey Charles Greenwald, Lipschitz spaces on the surface of the unit

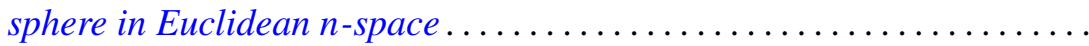

Herbert Paul Halpern, Open projections and Borel structures for

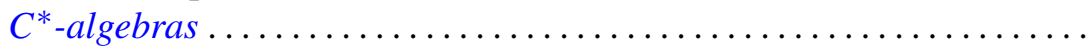

Frederic Timothy Howard, The numer of multinomial coefficients divisible

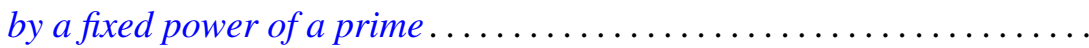

Lawrence Stanislaus Husch, Jr. and Ping-Fun Lam, Homeomorphisms of manifolds with zero-dimensional sets of nonwandering points........ 109

Joseph Edmund Kist, Two characterizations of commutative Baer rings ...

Lynn McLinden, An extension of Fenchel's duality theorem to saddle functions and dual minimax problems ................

Leo Sario and Cecilia Wang, Counterexamples in the biharmonic classification of Riemannian 2-manifolds...

Saharon Shelah, The Hanf number of omitting complete types ...

Richard Staum, The algebra of bounded continuous functions into a

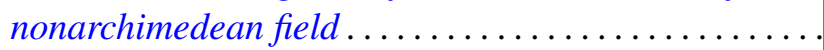

James DeWitt Stein, Some aspects of automatic continuity ..

Tommy Kay Teague, On the Engel margin

John Griggs Thompson, Nonsolvable finite groups all of whose local subgroups are solvable, $V \ldots \ldots \ldots \ldots \ldots \ldots \ldots \ldots$

Kung-Wei Yang, Isomorphisms of group extensions 